\title{
Color Doppler sonography of ovarian arteries as a tool in the differential diagnosis of pelvic inflammatory disease and appendicitis
}

Received: 16 August 2004/ Accepted: 28 November 2004/Published online: 18 February 2005

(C) Springer-Verlag Berlin / Heidelberg 2005

\begin{abstract}
The purpose of this study was to show whether color Doppler ultrasonography of the ovarian arteries is a useful tool for the differential diagnosis of pelvic inflammatory disease (PID) and appendicitis. The study included 50 female patients with clinical symptoms of acute pelvic inflammation and 50 healthy women of the same age as a control group. All were examined with color Doppler ultrasonography to visualize the internal genitalia and estimate the blood flow through the ovarian arteries. Peak systolic velocity (PSV), end diastolic velocity, pulsatility index (DPI), and resistance index were measured. In the healthy women, ovaries were normal in size, and the parameters of ovarian arterial flow were PSV $0.30-0.50$, systolic/diastolic ratio $<0.7$, DPI $1.23-1.50$. In 45 of the patients, there was infection and enlargement of the ovaries and/or salpinx, and flow parameters were PSV $0.40-0.70$, systolic/diastolic ratio $0.5-0.8$, and DPI 1.40 1.96. In five cases the ultrasound image was obscure, and flow parameters were similar to those of healthy women. These patients underwent surgery for a diagnosis of appendicitis. Our study showed that in cases of diagnostic differentiation between PID and appendicitis, examination with color Doppler ultrasound is very easy and useful. The relatively increased flow parameters in ovarian arteries direct the diagnosis to PID.
\end{abstract}

Keywords Pelvic inflammatory disease $\cdot$ Acute appendicitis · Color Doppler ultrasound

\section{Introduction}

Acute pelvic or lower abdominal pain in women is a symptom that commonly leads them to physicians. But

V. Panaritis $\cdot$ A. V. Kyriakidis $(\bowtie) \cdot$ M. Pyrgioti

A. Apostolopoulos

General Hospital of Amfissa, Amfissa Fokida, Greece

E-mail: alkidi@hotmail.com

Tel.: + 30-2265072265

Fax: + 30-2265022086 determining the cause of the pain may be difficult because many diseases present similarly. Pelvic infection presents a serious problem for the practicing physician and for the radiologist who is called to determine the origin and extent of the inflammatory process. Ultrasound has become a valuable primary imaging tool in assessing acute pelvic pain in women, both for diagnosis and for assessment of complications.

\section{Aim of study}

This study evaluated the use of color Doppler ultrasonography for differentiating pelvic inflammatory disease (PID) from other pelvic infections, mainly appendicitis.

\section{Patients and methods}

In this study, carried out over 1 year, we included 50 female patients who came to the emergency setting of our hospital with clinical symptoms of acute pelvic infection. Lower or diffuse abdominal pain was their main complaint. Their ages ranged from 13 to 30 years, with a mean age of 24 years. We excluded from our study those women who were in the first half of their menstrual cycles because in some previous measurements, we found that blood flow in the ovarian arteries increased during estrogen stimulation in the first half of the menstrual cycle. We also excluded women with menstrual cycle problems, such as amenorrhea or luteal phase defect; those with previous history of PID; those with polycystic ovaries; and those with positive pregnancy tests. The laboratory exams showed minimally elevated values of leukocytes, C-reactive protein, and erythrocyte sedimentation rate.

For a control group we examined 50 healthy women of the same age range with no clinical symptoms and with normal biochemical blood test examinations. These volunteers were selected from subjects visiting our hospital for a screening program/yearly gynecological 
examination who agreed to participate in this study after the purposes, risks, and potential benefits were explained to them.

All patients and healthy volunteers were examined with color Doppler ultrasonography to visualize the internal genitalia and estimate the blood flow through the ovarian arteries. Peak systolic velocity (PSV), end diastolic velocity, pulsatility index (DPI), and resistance index were measured.

Patient demographics were taken from the case notes. The two-sample $t$-test was used to assess differences in the mean values for age. The one-tailed test for proportions was used to confirm or refute any apparent differences in proportions (demographics, medical history between patients). The chi-square statistic was employed to assess the association between the groups of interest and the incidence of parameters of flow index values of the ovarian arteries. Values of $P<0.05$ were considered significant.

\section{Results}

In the healthy female volunteers, ovaries were normal in size, and the parameters of ovarian arterial flow were as follows:

PSV: $0.30-0.50$

Systolic/diastolic ratio: $<0.7$

DPI: $1.23-1.50$

In 45 of the patients, ultrasound showed infection and enlargement of the ovaries or salpinx. Flow parameters were as follows:

PSV: $0.40-0.70$

Systolic/diastolic ratio: $0.5-0.8$

DPI: $1.40-1.96$

In five cases there was a major diagnostic dilemma between appendicitis and PID. The boundaries of the right ovary were difficult to estimate, and flow parameters were similar to those of healthy women. In those doubtful cases, patients underwent surgery and appendectomy. Infection of the appendix was found, with its location following the route of the right ovary.

We also observed that by using Doppler examination with stable mechanical parameters, we could easily and clearly show the architecture of the ovarian microcirculation when infection was present.

\section{Discussion and conclusions}

Pelvic inflammatory disease is a major public health concern with significant medical and socioeconomic consequences. It is the most frequent cause of hospitalization among reproductive-age women and leads to infertility in about $30 \%$ of cases and ectopic pregnancies in $50 \%$ [1-3]. PID includes all the acute or chronic inflammations of the salpinx, ovaries, and, often, the nearby organs. It is a spectrum of upper genital tract inflammatory disorders that may include endometritis, salpingitis, tubo-ovarian abscess, and pelvic peritonitis [4]. The risk factors for PID include multiple sexual partners, a history of previous sexually transmitted infections/PID [4], lack of consistent condom use, and the use of intrauterine devices for contraception.

The principal symptom of PID is lower abdominal pain. Its intensity may vary. Usually it is continuous and bilateral with crampy exacerbations, often starting with the onset of menses and following it. Other symptoms include prolonged or increased menstrual bleeding, dysmenorrhea, dysuria, dyspareunia, and vaginal discharge. The patient may complain of right upper quadrant pain, which may be acute or severe as a result of perihepatitis (Fitz-Hugh-Curtis syndrome). Nausea and vomiting develop with severe PID, as does a corresponding peritonitis. Adnexal tenderness, uterine tenderness, and cervical motion tenderness may be noted during physical examination [4-7].

PID is sometimes difficult to diagnose. Its symptoms and signs are common for different diseases and vary from case to case. It can be overdiagnosed, leading to misdiagnosis of PID in healthy women, or underdiagnosed or misdiagnosed when women have other diseases, resulting in delayed appropriate treatment for the other conditions, including appendicitis. The differential diagnosis includes irritable bowel disease, diverticulitis, inflammatory bowel disease, adhesions after laparoscopy, endometriosis, and acute appendicitis [8].

Acute appendicitis is often misdiagnosed as PID and vice versa. Acute appendicitis usually presents with diffuse abdominal pain around the navel, which thereafter is located in the right lower quadrant. It is usually accompanied by nausea, vomiting, and a slightly elevated erythrocyte sedimentation rate, C-reactive protein, and leukocytes. Women with appendicitis more frequently have isolated right lower abdominal pain than do those with PID. On average, women with PID have pain twice as long as those with appendicitis. Compared with those with appendicitis, patients with PID are significantly less likely to report vomiting $[9,10]$.

Physicians face a common diagnostic dilemma when female patients present with lower abdominal pain. Early diagnosis is very important in order to provide the best treatment for the patient. Transabdominal and transvaginal ultrasound is a helpful diagnostic examination. It is possible to view the internal organs of the pelvic area to see whether the fallopian tubes are enlarged or whether an abscess is present. Laparoscopy may confirm the diagnosis [11, 12].

Color Doppler ultrasound is widely used nowadays for assessing differences in blood flow of the ovarian arteries. It has been used for the diagnosis of luteal phase defect, for hemodynamic assessment of PID, and for assessment of women with hypoestrogenic amenorrhea. It has also been used for the differential diagnosis of appendicitis and PID [13-16]. 
In our study, the pulsatility index of the ovarian artery was measured and compared with values obtained from healthy women. One of the major signs of inflammation is a change in vascular flow. It is possible to detect these changes with the help of transvaginal color Doppler velocimetry. We detected decreased vascular resistance in acute infection. Doppler velocity waveforms were quantitated by the pulsatility index. A low-resistance blood flow was found at the margin of the infectious complex. The severity of the infection as determined by $\mathrm{C}$-reactive protein values was inversely correlated to the pulsatility index. Angiogenesis is responsible for the increased flow.

We conclude that transvaginal color Doppler is a useful additional tool in diagnosing and treating patients with PID. Color Doppler ultrasound of the ovarian artery seems to be a reliable, easy, and quick method when a prompt acute diagnosis is needed.

The sonographic criteria consistent with acute appendicitis were clearly different from those of acute inflammatory disease. More prospective trials are needed to evaluate the impact of transvaginal color Doppler ultrasound in the differential diagnosis between appendicitis and pelvic inflammatory disease.

\section{References}

1. Mathias SD, Kuppermann M, Liberman RF, et al. (1996) Chronic pelvic pain: prevalence, health-related quality of life and economic correlates. Obstet Gynecol 87:321-327

2. Jamieson DJ, Steege JF (1996) The prevalence of dysmenorrhea, dyspareunia, pelvic pain and irritable bowel syndrome in primary care practices. Obstet Gynecol 87:55-58
3. Cromwell P, Riser W, Risser J (2000) Prevalence and incidence of pelvic inflammatory disease in incarcerated adolescents. Sex Transm Dis 28:392-396

4. Westrom L, Eschenbach D (1998) Pelvic inflammatory disease. In: Holmes K, Sparling P, Mardh P, et al. (eds) Sexually transmitted diseases. McGraw-Hill, New York, pp 783-810

5. McCormack WM (1994) Pelvic inflammatory disease. N Engl J Med 330:115-119

6. Soper DE (1994) Pelvic inflammatory disease. Infect Dis Clin North Am 8:821-840

7. Centers for Disease Control (1998) Guidelines for treatment of sexually transmitted diseases. MMWR. Recommendations and reports, vol. 47, no. RR-1

8. Ross J (2001) Pelvic inflammatory disease. BMJ 322:658-659

9. Molander P, Paavonen J, Sjoberg J, Savelli L, Cacciatore B (2002) Transvaginal sonography in the diagnosis of acute appendicitis. Ultrasound Obstet Gynecol 20:496-501

10. Webster DP, Schneider CN, Cheche S, Daar AA, Miller G (1993) Differentiating acute appendicitis from pelvic inflammatory disease in women of childbearing age. Am J Emerg Med 11:569-572

11. Bau A, Arti M (2000) Acute pelvic pain: ultrasound evaluation. Semin Ultrasound CT MR 21:78-93

12. Ghiatas A (2004) The spectrum of pelvic inflammatory disease. Eur Radiol 14:E184-E192

13. Papadimitriou A, Kalogirou D, Antoniou G, Petridis N, Kalogirou O, Kalovidouris A (1996) Power Doppler ultrasound: a potentially useful alternative in diagnosing pelvic pathologic conditions. Clin Exp Gynecol 23:229-232

14. Pellizzari P, Esposito C, Siliotti F, Marchiori S, Gangemi M (2002) Colour Doppler analysis of ovarian and uterine arteries in women with hypoestrogenic amenorrhea. Hum Reprod $17: 3208-3212$

15. Alatas C, Aksoy E, Akarsu C, Yakin K, Bahceci M (1996) Hemodynamic assessment in pelvic inflammatory disease by transvaginal color Doppler ultrasonography. Eur J Obstet Gynecol Reprod Biol 70:75-78

16. Kalogirou D, Antoniou G, Botsis D, Kontovardis A, Vitoratos N, Giannikos L (1997) Transvaginal Doppler ultrasound with color flow imaging in the diagnosis of luteal phase defect (LPD). Clin Exp Obstet Gynecol 24:95-97 